\title{
Tinea Capitis in Campania: A 5-Year Retrospective Study in Childhood and Adolescence (2013-2018)
}

\author{
Eleonora Cinelli Alessia Villani Gabriella Fabbrocini Massimiliano Scalvenzi \\ Lucia Gallo
}

Section of Dermatology, University of Naples Federico II, Naples, Italy

\section{Keywords}

Tinea capitis · Hair · Fungi · Microscopy

\begin{abstract}
Introduction: Tinea capitis is a dermatophytic infection of the scalp hair commonly affecting children in school age. Methods: Here we report our data of a 5-year retrospective study, including all patients between 1 and 18 years old referred to our Mycological Laboratory for suspected tinea capitis. Results: A total of 202 patients were included and positive tests for tinea capitis were found in 86/202 (42.57\%) patients. Most of the patients, $126 / 202$ (62.38\%), had not applied any topical therapy or taken any systemic drug, whereas 76/202 (37.62\%) had already treated the disease. Discussion: We underline the importance of the differential diagnosis of scalp lesions, the possibility of the so-called "tinea incognita" in some cases, and the species isolated in fungal cultures. A high number of Trichophyton mentagrophytes were isolated $(71 / 86 ; 82 \%)$ and this phenomenon may be due to a particular suspected clinical picture of tinea capitis that induced the dermatologist to ask for a more detailed exam.

(c) 2019 S. Karger AG, Basel
\end{abstract}

\section{Introduction}

Tinea capitis (TC) is a dermatophytic infection of the scalp hair commonly affecting children in school age. It is usually caused by anthropophilic or zoophilic dermatophytes of the genera Microsporum and Trichophyton that invade keratinized tissues and so, infect the scalp [1]. Although Microsporum canis represents the main cause of TC in Europe, anthropophilic agents of TC have become more prevalent in the United Kingdom, probably because of the recent immigration and travel configuration $[2,3]$. The last retrospective study performed in Campania reported 143 cases of TC in a group of patients aged $<18$ years; $M$. canis was the organism most frequently isolated $(64.1 \%)$, confirming the general European trend [4]. Herein, we report our data about the incidence of TC, based on a 5-year retrospective study, including all patients between 1 and 18 years of age that were referred to our Mycological Laboratory for suspected TC.

\section{Material and Methods}

This study was conducted at the Mycology Laboratory of the University of Naples "Federico II" between January 2013 and September 2018. All patients aged $<18$ years with suspected TC were included. Age, sex, time of appearance of the lesions, previous

\section{KARGER}

E-Mail karger@karger.com

www.karger.com/sad
Alessia Villani

Department of Dermatology, University of Naples Federico II

Via Pansini 5

IT-80131 Naples (Italy)

E-Mail ali.vil@hotmail.it 
Table 1. Data on previous topical and systemic therapies

\begin{tabular}{lc}
\hline No previous therapies & $126 / 202(62.38 \%)$ \\
Previous therapies & $76 / 202(37.62 \%)$ \\
Topical and systemic antimycotics & $32 / 76(42.11 \%)$ \\
Topical corticosteroids & $13 / 76(17.11 \%)$ \\
Systemic antimycotics & $13 / 76(17.11 \%)$ \\
Topical antimycotics & $6 / 76(7.89 \%)$ \\
Systemic antibiotics & $2 / 76(2.63 \%)$ \\
Topical antimycotics and antihistaminics & $2 / 76(2.63 \%)$ \\
Topical and systemic antibiotics & $2 / 76(2.63 \%)$ \\
Topical antibiotics & $2 / 76(2.63 \%)$ \\
Topical corticosteroids and antibiotics & $1 / 76(1.31 \%)$ \\
Topical antimycotics and antibiotics & $1 / 76(1.31 \%)$ \\
Topical corticosteroids and antimycotics & $1 / 76(1.31 \%)$ \\
Topical cholecalciferol & $1 / 76(1.31 \%)$ \\
\hline
\end{tabular}

therapies, and comorbidities were registered for each patient and samples of the scalp hair, including both scaly material from cutaneous scraping and plucked hair, were collected. Potassium hydroxide $20 \%$ was used to prepare specimens for microscope examination; furthermore, examination of samples that were contaminated by a high number of bacteria was repeated after 5 days of topical disinfectant treatment of the suspicious lesion. Microscope examination was considered positive in the presence of spores (both ectothrix and endothrix), hyphae, arthroconidia/conidia or trichospores. For each positive result, part of the sample was directly inoculated in Sabouraud dextrose agar + chloramphenicol + gentamicin culture media and incubated for 14 days at $27^{\circ} \mathrm{C}$ to make fungal culture tests. Cultures not showing noticeable fungal colonies were incubated for an additional week before they were considered negative and each positive colonial morphology was detected with microscopic evaluation.

\section{Results}

The majority of patients, $126 / 202(62.38 \%)$, had not applied any topical therapy or taken any systemic drug, whereas $76 / 202(37.62 \%)$ had already treated the disease (Table 1$)$. Only 5 patients $(2.48 \%)$ presented comorbidities: psoriasis (2 patients), atopic dermatitis (1), allergic contact dermatitis (1), and diabetes (1). Positive tests for TC were found in 86 (42.57\%; 48 males and 38 females) patients. The following species resulted from the fungal cultures performed: 72/86 (82\%) T. mentagrophytes, 7/86 (9\%) M. canis, and 7/86 (9\%) T. rubrum.

\section{Discussion}

Our data are based on a 5-year retrospective study, involving patients aged $<18$ years with suspected TC. 86 patients were positive with a male prevalence $(48 / 86)$, be- ing in prepuberal age, confirming what had been reported in the literature [2]. Although this article highlights the importance of considering all possible differential diagnoses of scalp lesions, as the majority of patients had a negative result $(116 / 202,57.43 \%)$ for fungi, TC still remains a challenge in the clinical practice and fungal tests should always be considered to exclude fungal infections. Infectious and nonmimicking diseases in prepuberal/adolescent patients, ranging from alopecia areata to bacterial infection, should be considered as possible differential diagnosis [5]. Nowadays, the increasing use of trichoscopy helps the clinician in the diagnosis, as confirmed by a recent study that has pointed out how a distinct trichoscopic pattern characterized by perifollicular scaling with dystrophic or broken hair could be recognized in patients affected by TC [6]. Furthermore, it has been reported that comma hairs, corkscrew hairs, zigzag hairs, and whitish sheath are significantly more frequent in patients with a positive mycological culture. In particular, comma hairs have been described to be more frequent in patients with TC positive for Trycophyton, and zigzag hairs are more frequent in patients positive for Microsporum [6]. Interestingly, a significant group of positive patients $(12 / 86$, $13.95 \%)$ had already been treated with nonantimycotic topical or systemic therapy, without performing microscopical or cultural examination, inducing the so-called "tinea incognita." On the other hand, a group of patients without a confirmed diagnosis of TC $(21 / 116,18.10 \%)$ had already taken systemic or topical antimycotic therapy. This phenomenon could have led to a change in the clinical picture and a diagnostic delay. Further studies should focus on the origin of these inappropriate treatments to understand whether it is due to self-medication of the patient or to an incorrect diagnosis helping us to understand the burden of incorrect self-medication of the patients and so, promoting correct information of patients. A limitation of our study was the lack of data on the counterpart (the non-TC group) and further studies are required to add data on the different scalp diseases diagnosed. Furthermore, we noticed a high number of isolated T. mentagrophytes; these results are in contrast with a previous study conducted in Campania that confirmed the prevalence of $M$. canis [4]. The higher number of positive cultures for T. mentagrophytes may be due to a particular suspected clinical picture of TC that induced the dermatologist to ask for a more detailed exam. Moreover, identifying the correct pathogenic species would be important to choose a correct treatment based on the specific fungal species [7]. Finally, our study shows the incidence of TC in Campania, Italy, highlighting a high per- 
centage of the Trichophyton genera isolated in our cultures. This is not in line with the general European trend that reported $M$. canis as the causative agent most frequently isolated. The major incidence of Trichophyton could be attributable and claimed to be due to the immigration state (from being endemic to being present across the globe), which facilitates the contagion flow. Nevertheless, future studies and surveys on a larger group of patients are required to better define the shift of infectious species implied in TC and the possible associated factors, such as exposure to animals or travels.

\section{Statement of Ethics}

Informed consent was obtained from the patient for inclusion in the study.

\section{Disclosure Statement}

The authors have no conflict of interest to disclose.

\section{References}

1 Kieliger S, Glatz M, Cozzio A, Bosshard PP. Tinea capitis and tinea faciei in the Zurich area - an 8-year survey of trends in the epidemiology and treatment patterns. J Eur Acad Dermatol Venereol. 2015 Aug;29(8):1524-9.

2 Fuller LC, Barton RC, Mohd Mustapa MF, Proudfoot LE, Punjabi SP, Higgins EM. British Association of Dermatologists' guidelines for the management of tinea capitis 2014. Br J Dermatol. 2014 Sep;171(3):454-63.
3 Borman AM, Campbell CK, Fraser M, Johnson EM. Analysis of the dermatophyte species isolated in the British Isles between 1980 and 2005 and review of worldwide dermatophyte trends over the last three decades. Med Mycol. 2007 Mar; 45(2):131-41.

4 Calabrò G, Patalano A, Fiammenghi E, Chianese C. Tinea capitis in Campania, Italy: a 9-year retrospective study. G Ital Dermatol Venereol. 2015 Aug;150(4):363-7.
5 Shastry J, Ciliberto H, Davis DM. Tinea capitis mimicking dissecting cellulitis in three children. Pediatr Dermatol. 2018 Jan; 35(1):e79-83.

6 Brasileiro A, Campos S, Cabete J, Galhardas C, Lencastre A, Serrão V. Trichoscopy as an additional tool for the differential diagnosis of tinea capitis: a prospective clinical study. $\mathrm{Br}$ Dermatol. 2016 Jul;175(1):208-9.

7 Gupta AK, Summerbell RC. Tinea capitis. Med Mycol. 2000 Aug;38(4):255-87. 